\title{
Long non-coding RNA DUXAP8 promotes tumorigenesis by regulating IGF1R via miR-9-3p in hepatocellular carcinoma
}

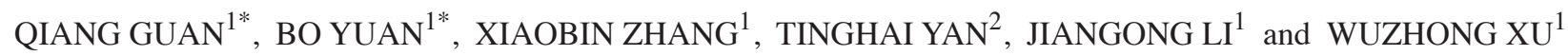 \\ ${ }^{1}$ Department of Hepatobiliary Surgery, People's Hospital of Dongying, Dongying, Shandong 257091; \\ ${ }^{2}$ Department of Oncology, People's Hospital of Wudi, Binzhou, Shandong 251900, P.R. China
}

Received July 30, 2019; Accepted August 13, 2020

DOI: $10.3892 /$ etm.2021.10187

\begin{abstract}
Hepatocellular carcinoma (HCC) is the third leading cause of cancer-related death worldwide with a low 5-year survival rate. Long non-coding RNA (lncRNA) double homeobox A pseudogene 8 (DUXAP8) is an oncogene and a potential biomarker in various tumors, such as ovarian, colorectal and non-small-cell lung cancer. However, the function and molecular mechanism underlying DUXAP8 in HCC progression is not completely understood. The expression of DUXAP8, microRNA (miR)-9-3p and insulin-like growth factor 1 receptor (IGF1R) in HCC tissues and cells was detected via reverse transcription-quantitative PCR. The expression levels of IGF1R and epithelial-mesenchymal transition-associated proteins (Snail, Slug, E-cadherin, N-cadherin and vimentin) were assessed via western blotting. The effects of DUXAP8, miR-9-3p and IGF1R on proliferation, migration and invasion were examined by conducting Cell Counting Kit-8 and Transwell assays, respectively. The interaction between miR-9-3p and DUXAP8 or IGF1R was predicted using StarBase or TargetScan, and further assessed using dual luciferase reporter and RNA immunoprecipitation assays. DUXAP8 and IGF1R were upregulated and miR-9-3p was downregulated in HCC tissues and cells compared with adjacent healthy tissues and a normal liver cell line, respectively. miR-9-3p overexpression decreased the protein expression level of IGF1R, and miR-9-3p knockdown enhanced the protein expression level of IGF1R in HCC cells compared with the corresponding control groups. Moreover, compared with the corresponding control groups, DUXAP8 knockdown and miR-9-3p overexpression increased E-cadherin protein
\end{abstract}

Correspondence to: Dr Wuzhong Xu, Department of Hepatobiliary Surgery, People's Hospital of Dongying, 317 South Road, Dongying, Shandong 257091, P.R. China

E-mail: xwzhong1963@163.com

*Contributed equally

Key words: hepatocellular carcinoma, long non-coding RNA double homeobox A pseudogene 8, microRNA-9-3p, insulin-like growth factor 1 receptor, proliferation, migration, invasion, epithelial-mesenchymal transition expression levels, and decreased Snail, Slug, N-cadherin and vimentin protein expression levels. However, miR-9-3p inhibitor and IGF1R overexpression reversed DUXAP8 knockdown- and miR-9-3p overexpression-induced effects, respectively. In addition, compared with the corresponding control groups, DUXAP8 knockdown and miR-9-3p overexpression suppressed proliferation, migration and invasion, which was reversed by miR-9-3p inhibitor and IGF1R overexpression, respectively. Moreover, miR-9-3p as the target of DUXAP8 and IGF1R as the target of miR-9-3p were verified in HCC cells. IncRNA DUXAP8 contributed to HCC tumorigenesis via the miR-9-3p/IGF1R axis, providing a novel therapeutic approach for HCC diagnosis and treatment.

\section{Introduction}

Hepatocellular carcinoma (HCC) is a main type of primary malignant tumor and is the third leading cause of cancer-related death worldwide $(1,2)$. Despite advances in diagnosis and treatment, the long-term survival rate of patients with HCC remains poor ( $\sim 5$ years) due to the early metastasis and high recurrence rate (3). Therefore, identifying a novel molecular mechanism and developing effective therapeutic strategies for HCC is important.

Long non-coding RNAs (lncRNAs) are transcripts that are $>200$ nucleotides in length and lack protein coding capacity (4). Increasing evidence has suggested that IncRNAs serve critical functions in diverse biological and cellular processes, such as growth, invasion, metastasis, differentiation and tumorigenesis (5-8). The dysregulation of lncRNAs has been reported to be closely associated with numerous tumors, including HCC (9). For example, Li et al (10) reported that lncRNA focally amplified long non-coding RNA in epithelial cancer (FAL1) was upregulated in HCC, and FAL1 overexpression promoted proliferation and metastasis via regulating microRNA (miRNA/miR)-1236 expression. Ma et al (11) demonstrated that lncRNA CDKN2B antisense RNA 1 knockdown inhibited cell proliferation, metastasis and invasion via competitively binding to miR-122-5p in HCC. Double homeobox A pseudogene 8 (DUXAP8) is a pseudogene-derived lncRNA that maps to chromosome 20q11, and has been reported to be an oncogene and a potential biomarker in various tumors $(12,13)$. Moreover, previous reports have also verified that DUXAP8 overexpression induced cell proliferation and migration in 
renal cell carcinoma (14) and non-small-cell lung cancer (15). However, the function and molecular mechanism underlying DUXAP8 in HCC progression is not completely understood.

miRNAs, a class of short non-coding RNAs, are involved in the regulation of protein-coding genes by suppressing mRNA translation (16). Previous studies have demonstrated that miRNAs could exert a tumor suppressor role in various types of cancer. For instance, miR-574-3p has been indicated to inhibit the malignant behavior of liver cancer cells via interacting with disintegrin and metalloproteinase domain-containing protein 28 (17). Moreover, miR-27b-5p has been reported to repress the growth and metastasis of ovarian carcinoma cells by targeting C-X-C motif chemokine 1 (18). In addition, the suppressive effect of miRNAs, such as miR-188-5p and miR-1271, on the progression of HCC has also been demonstrated in previous studies $(19,20)$. Furthermore, a previous study indicated that miR-9-3p served as a tumor suppressor and constrained cell proliferation by targeting tafazzin expression in HCC cells (21). However, the mechanism underlying miR-9-3p in HCC is not completely understood.

Insulin-like growth factor 1 receptor (IGF1R) has tyrosine kinase activity and has been demonstrated to function as an antiapoptotic agent by enhancing cell survival in a variety of different types of cancer $(22,23)$. For example, knockdown of IGF1Rhas been indicated to decrease cell proliferation, migration, and invasion in ovarian cancer cells (24). Similarly, downregulation of IGF1R has been revealed to suppress cell growth and improve cisplatin sensitivity of head and neck squamous cell carcinoma cells (25). Moreover, some studies reported that IGF1R was upregulated, and promoted proliferation, migration, invasion and EMT in HCC (26-30). The results suggested that IGF1R served important roles in HCC progression.

Therefore, the aim of the present study was to investigate the function of DUXAP8 and to explore whether the involvement of DUXAP8 in HCC was mediated via the miR-9-3p/IGF1R axis.

\section{Materials and methods}

Clinical specimens and cell culture. HCC pathologically confirmed tumor tissues and adjacent healthy tissues $(>5 \mathrm{~cm}$ from the tumor margin) were obtained from 38 patients with HCC (31-85 years old) who had undergone surgical treatment at People's Hospital of Dongying between January 2011 and June 2014. The present study was approved by People's Hospital of Dongying Ethics Committee (Dongying, China). Written informed consent was obtained from all patients.

Human normal liver cell line (THLE-2) and HCC cell lines (SK-HEP-1 and Hep3B) were purchased from the American Type Culture Collection. HCC cell lines (Huh-7 and Huh-1) were purchased from the Japanese Collection of Research Bioresources Cell Bank. All cells were maintained at $37^{\circ} \mathrm{C}$ with $5 \% \mathrm{CO}_{2}$ in DMEM (Gibco; Thermo Fisher Scientific, Inc.) supplemented with $10 \%$ FBS (Invitrogen; Thermo Fisher Scientific, Inc.).

Cell transfection. DUXAP8 small interfering (si)RNA (si-DUXAP8\#1, 5'-AGT TCAGTGTATTTGATAATA-3'; si-DUXAP8\#2, 5'-GATTTGGTTTCAGAATCAAAG-3';
si-DUXAP8\#3, 5'-GATGGTGTTGTACCACCTATA-3') and scramble siRNA control [si-negative control (NC): 5'-TTCTCC GAACGTGTCACGTTT-3'] were purchased from Shanghai GenePharma Co., Ltd. miR-9-3p mimic (5'-ATAAAGCTA GATAACCGAAAGT-3'), scramble mimic control (miR-NC; 5'-TTCTCCGAACGTGTCACGTTT-3'), miR-9-3p inhibitor (5'-ACTTTCGGTTATCTAGCTTTAT-3') and scramble inhibitor control (anti-miR-NC; 5'-CAGTACTTTTGTGTAGT ACAA-3') were purchased from Applied Biosystems; Thermo Fisher Scientific, Inc. Furthermore, to overexpress DUXAP8 or IGF1R, the full length sequence of DUXAP8 or IGF1R was inserted into the pcDNA3.1 vector (Invitrogen; Thermo Fisher Scientific, Inc.) to generate pcDNA3.1-DUXAP8 (DUXAP8) and pcDNA3.1-IGF1R (IGF1R), respectively. pcDNA3.1 empty vector was used as negative control. SK-HEP-1 and Huh-7 cells in 6 -well plates $\left(2 \times 10^{5}\right.$ cells/well $)$ were transfected with oligonucleotides $(50 \mathrm{nM})$ and plasmids $(2 \mu \mathrm{g})$ using Lipofectamine $^{\circledR} 2000$ (Invitrogen; Thermo Fisher Scientific, Inc.). Following incubation for $48 \mathrm{~h}$, the transfected cells were harvested and utilized for subsequent experiments.

$R N A$ extraction and reverse transcription-quantitative PCR $(R T-q P C R)$. Total RNA was extracted from clinical tissues and HCC cells using TRIzol ${ }^{\circledR}$ reagent (Invitrogen; Thermo Fisher Scientific, Inc.). Subsequently, total RNA was reverse transcribed into cDNA using M-MLV reverse transcriptase (Thermo Fisher Scientific, Inc.) including 5x buffer, $10 \mathrm{mM}$ dNTPs, and $50 \mu \mathrm{mol}$ oligo dT primers (cat. no. 18418012; Thermo Fisher Scientific, Inc.). The RT reaction was performed at $70^{\circ} \mathrm{C}$ for $10 \mathrm{~min}$ followed by $42^{\circ} \mathrm{C}$ for $1 \mathrm{~h}$, according to the manufacturer's protocol. DUXAP8 and IGF1R mRNA expression levels were measured via qPCR using SYBR Premix Ex Taq (Takara Biotechnology Co., Ltd.). The thermocycling conditions were as follows: Denaturation at $95^{\circ} \mathrm{C}$ for $10 \mathrm{~min}$, followed by 40 cycles of denaturation at $95^{\circ} \mathrm{C}$ for $30 \mathrm{sec}$, annealing at $60^{\circ} \mathrm{C}$ for $30 \mathrm{sec}$ and extension at $72^{\circ} \mathrm{C}$ for 1 min. Has-miR-9-3p-specific TaqMan primer (5'-ATAAAGCTAGATAACCGAAAGT-3') was purchased from Applied Biosystems (cat. no. 4427975; Thermo Fisher Scientific, Inc.; Assay ID, 002231) for synthesis of cDNA. miR-9-3p expression levels were measured via qPCR using a TaqMan MicroRNA Assay Kit (Applied Biosystems; Thermo Fisher Scientific, Inc.). The thermocycling conditions were as follows: $16^{\circ} \mathrm{C}$ for $30 \mathrm{~min}, 42^{\circ} \mathrm{C}$ for $30 \mathrm{~min}, 85^{\circ} \mathrm{C}$ for $5 \mathrm{~min}$ and $4^{\circ} \mathrm{C}$ hold. The following primers were used for qPCR: DUXAP8 forward, 5'-AGACGCCATGGAACAT-3' and reverse, 5'-AAG CGGAGACCTGAGGAG-3'; GAPDH forward, 5'-AGAAGG CTGGGGCTCATTTG-3' and reverse, 5'-AGGGGCCAT CCACAGTCTTC-3'; IGF1R forward, 5'-TTTCCCACAGCA GTCCACCTC-3' and reverse, 5'-AGCATCCTAGCCTTC TCACCC-3'; miR-9-3p forward, 5'-TCTTTGGTTATCTAG CTGTAT-3' and reverse, 5'-GAACATGTCTGCGTATCT C-3'; and U6 forward, 5'-GCTTCGGCAGCACATATACTA AAA-3' and reverse, 5'-CGCTTCACGAATTTGCGTGTC AT-3'. Moreover, miRNA and mRNA expression levels were quantified using the $2^{-\Delta \Delta \mathrm{Cq}}$ method (31) and normalized to the internal reference genes U6 and GAPDH, respectively.

Cell proliferation assay. Cell proliferation was detected by performing a Cell Counting Kit-8 (CCK-8) assay (Beyotime 
Institute of Biotechnology) according to the manufacturer's protocol. Briefly, transfected SK-HEP-1 and Huh-7 cells $\left(2.5-5 \times 10^{3}\right.$ cells/well) were inoculated into 96 -well plates and incubated for $48 \mathrm{~h}$ at $37^{\circ} \mathrm{C}$. Subsequently, CCK-8 $(10 \mu \mathrm{l})$ solution was added to each well and cultured at $37^{\circ} \mathrm{C}$ for $0,24,48$ or $72 \mathrm{~h}$. The optical density value of each well was measured at a wavelength of $450 \mathrm{~nm}$ using a Varioskan Flash Microplate Reader (Thermo Fisher Scientific, Inc.).

Cell migration and invasion assays. Cell migration and invasion were detected using 24-well Transwell chambers (BD Biosciences) according to the manufacturer's protocol. Transfected SK-HEP-1 and Huh-7 cells were resuspended in serum-free DMEM overnight. Subsequently, cells $\left(5 \times 10^{4}\right)$ were plated into the upper chamber, which was pre-coated with Matrigel ${ }^{\circledR}$ at $4^{\circ} \mathrm{C}$ overnight and subsequently maintained at $37^{\circ} \mathrm{C}$ for $4-5 \mathrm{~h}$ (BD Biosciences) for the cell invasion assay. DMEM supplemented with 10\% FBS (Invitrogen; Thermo Fisher Scientific, Inc.) was plated in the lower chamber. Following incubation for $24 \mathrm{~h}$ at $37^{\circ} \mathrm{C}$, cells in the upper chamber were removed using cotton swabs. Migratory or invading cells were fixed using methanol for $30 \mathrm{~min}$ at $4^{\circ} \mathrm{C}$ and stained with $0.1 \%$ crystal violet solution for $20 \mathrm{~min}$ at $37^{\circ} \mathrm{C}$, followed by observation using an inverted fluorescence microscope (magnification, x100; Nikon Corporation).

Western blotting. Western blotting was performed to measure the protein expression levels of IGF1R and EMT-related proteins (E-cadherin, N-cadherin and vimentin) in SK-HEP-1 and Huh-7 cells. Total protein was isolated from cells using pre-cold RIPA buffer (Beyotime Institute of Biotechnology) including protease inhibitor. Total protein was quantified using a BCA protein assay kit (Pierce; Thermo Fisher Scientific, Inc.). Proteins $(50 \mu \mathrm{g})$ were separated via $10 \%$ SDS-PAGE and electrotransferred to nitrocellulose membranes (EMD Millipore). The membranes were blocked with 5\% non-fat milk at room temperature for $1 \mathrm{~h}$. Subsequently, the membranes were incubated overnight at $4{ }^{\circ} \mathrm{C}$ with primary antibodies targeted against: IGF1R (1:1,000; cat. no. 3027), Snail (1:1,000; cat. no. 3879), Slug (1:1,000; cat. no. 9585), E-cadherin (1:1,000; cat. no. 14472), N-cadherin (1:500; cat. no. 14215), vimentin (1:1,000; cat. no. 5741) and GAPDH (1:1,000; cat. no. 5174; all from Cell Signaling Technology, Inc.). The membranes were incubated with a horseradish peroxidase-labeled anti-rabbit IgG secondary antibody (1:1,000; sc-2027; Santa Cruz Biotechnology, Inc.) at room temperature for $1 \mathrm{~h}$. Protein bands were visualized using an ECL detection kit (Pierce; Thermo Fisher Scientific, Inc.) and analyzed with Quantity One v4.6.2 software (Bio-Rad Laboratories, Inc.). GAPDH was used as the loading control.

Dual-Luciferase reporter assay. The target gene of DUXAP8 or miR-9-3p was predicted using StarBase v2.0 (http://starbase.sysu.edu.cn/starbase2/) and TargetScan (www.targetscan.org) online prediction software, respectively. Based on the bioinformatics prediction, a partial DUXAP8 fragment containing the wild-type (WT) or mutant (MUT) miR-9-3p binding site, or the IGF1R 3'untranslated region (UTR) sequence containing the WT or MUT miR-9-3p binding site were amplified from HCC cells via PCR with 1 unit AmpliTaq DNA polymerase (Thermo Fisher Scientific, Inc.) using the following thermo cycling conditions: Pre-denaturation at $94^{\circ} \mathrm{C}$ for $10 \mathrm{~min} ; 30$ cycles at $94^{\circ} \mathrm{C}$ for $30 \mathrm{sec}, 53-57^{\circ} \mathrm{C}$ for $30 \mathrm{sec}$ and $72^{\circ} \mathrm{C}$ for $45 \mathrm{sec}$; and final extension at $72^{\circ} \mathrm{C}$ for $10 \mathrm{~min}$ ). The PCR product was subsequently cloned into the psiCHECK-2 vector (Promega Corporation) using the restriction endonuclease cleavage sites of XhoI and NotI (Promega Corporation). The following primers were used: DUXAP8-WT forward, 5'-CCGCTCGAGAACACTAATTGTAGACTATG-3' and reverse, 5'-ATAAGAATGCGGCCGCTATTGATGAGG ATTTTCAAT-3'; DUXAP8-MUT forward, 5'-TCAAAA CCCAGAAAACCATACAAGGACGATTTGTGA-3' and reverse, 5'-A ACCCAGAA AACCATACAAGGACGATT TGTGAATT-3'; IGF1R-WT forward, 5'-CCGCTCGAGTAG TCAGTTGACGAAGATCT-3' and reverse, 5'-ATAAGA ATGCGGCCGCTAGCTACACTCCAAAGGGAA-3'; and IGF1R-MUT forward, 5'-TTAGGACACCTGTTTACTAGA GGAACCGCAAATATGC-3' and reverse, 5'-GACACCTGT TTACTAGAGGAACCGCAAATATGCCAA-3'. SK-HEP-1 and Huh-7 cells in 24 -well culture plates $\left(2 \times 10^{5}\right.$ cells/well) were co-transfected using Lipofectamine 2000 (Invitrogen; Thermo Fisher Scientific, Inc.) with $100 \mathrm{ng}$ of DUXAP8-WT, DUXAP8-MUT, IGF1R-WT or IGF1R-MUT and $100 \mathrm{nM}$ miR-9-3p mimic or miR-NC. Following incubation for $48 \mathrm{~h}$, luciferase activities were assessed using a Dual-Luciferase reporter assay kit (cat. no. E1910; Promega Corporation), according to the manufacturer's protocol. Firefly luciferase activity was normalized to that of Renilla luciferase.

Bioinformatics analysis. The StarBase Pan-Cancer platform (http://starbase.sysu.edu.cn/panCancer.php) was used to analyze the correlation between DUXAP8, miR-9-3p and IGF1R in liver hepatocellular carcinoma (LIHC) $(32,33)$.

RNA immunoprecipitation (RIP) assay. To investigate the relationship between DUXAP8 and miR-9-3p, RIP was performed using the Magna RIP RNA-Binding Protein Immunoprecipitation kit (EMD Millipore) according to the manufacturer's protocol. Briefly, at $80 \%$ confluence, SK-HEP-1 and Huh-7 cells were harvested and lysed in RIP lysis buffer (EMD Millipore). Subsequently, $100 \mu$ l cell extract was incubated with RIP buffer including magnetic beads conjugated with anti-Argonaute2 (Ago2) antibody (1:1,000; cat. no. 03-248) or normal mouse IgG antibody $(1: 1,000$, cat. no. 12-371; both from EMD Millipore) overnight at $4^{\circ} \mathrm{C}$. Proteinase K (Invitrogen; Thermo Fisher Scientific, Inc.) was applied to digest the samples for $30 \mathrm{~min}$ at $55^{\circ} \mathrm{C}$, and the isolated RNAs were utilized for RT-qPCR analysis of DUXAP8 and miR-9-3p expression levels.

Statistical analysis. All statistical analyses were performed using SPSS software (version 18; SPSS, Inc.). Data are presented as the mean \pm SD. The $\chi^{2}$ test was used to analyze the association between DUXAP8 expression levels and clinicopathological features in HCC. Overall survival rates were evaluated using the Kaplan-Meier method with the long rank test applied for comparisons. The relationship among DUXAP8, miR-9-3p and IGF1R was analyzed using Pearson's correlation coefficient. Comparisons between two groups 
were analyzed using a paired Student's t-test. Comparisons among multiple groups were analyzed using one-way ANOVA followed by Tukey's post hoc test. $\mathrm{P}<0.05$ was considered to indicate a statistically significant difference. Experiments were repeated at least three times.

\section{Results}

DUXAP8 expression is upregulated in HCC tumor tissues and cells. To investigate the function of DUXAP8 in HCC, its expression was detected via RT-qPCR. Compared with adjacent healthy tissues, the expression of DUXAP8 was significantly increased in 38 HCC tumor tissues (Fig. 1A). DUXAP8 expression levels were also significantly elevated in HCC cell lines (SK-HEP-1, Huh-7, Hep38 and Huh-1) compared with the human liver cell line (THLE-2; Fig. 1B), with the highest expression levels observed in SK-HEP-1 and Huh-7 cells. Therefore, SK-HEP-1 and Huh-7 cells were selected for subsequent experiments. The association between DUXAP8 expression and clinicopathological factors in the 38 patients with HCC are presented in Table I. DUXAP8 expression was significantly associated with tumor size, TNM stage and metastasis $(\mathrm{P}<0.05)$, but was not significantly associated with age, sex, HbsAg and cirrhosis. Moreover, the overall survival curves suggested that DUXAP8 expression was inversely associated with the prognosis of patients with HCC (Fig. 1C). The results indicated that DUXAP8 may serve as a novel biomarker for the prognosis of HCC.

DUXAP8 induces HCC cell proliferation, migration, invasion and EMT. To assess the function of DUXAP8 in HCC cells, DUXAP8 knockdown was performed. The expression of DUXAP8 was significantly decreased in si-DUXAP8-transfected SK-HEP-1 and Huh-7 cells compared with si-NC-transfected cells (Fig. 2A). Since si-DUXAP8\#1 exhibited the highest knockdown inefficiency, it was selected for subsequent experiments. Therefore, the role of DUXAP8 in proliferation, migration, invasion and EMT was investigated by knocking down DUXAP8 expression in HCC cells. The CCK-8 and Transwell assay results suggested that DUXAP8 knockdown significantly inhibited SK-HEP-1 and Huh-7 cell proliferation (Fig. 2B and C), migration (Fig. 2D) and invasion (Fig. 2E) compared with si-NC. Subsequently, the effect of DUXAP8 on HCC cell EMT was investigated. The western blotting results indicated that DUXAP8 knockdown significantly increased E-cadherin protein expression levels, but significantly decreased Snail, Slug, N-cadherin and vimentin protein expression levels compared with si-NC (Fig. 2F), which suggested that DUXAP8 promoted SK-HEP-1 and Huh-7 cell EMT. Collectively, the results suggested that DUXAP8 enhanced HCC cell proliferation, migration, invasion and EMT.

miR-9-3p is a direct target of DUXAP8 in HCC cells. Increasing evidence has indicated that lncRNAs could exert their role by interacting with miRNAs (34-36). Therefore, bioinformatics-based target prediction analysis using StarBase was performed to identify the target miRNAs of DUXAP8. miR-9-3p was found to bind with DUXAP8 (Fig. 3D). To further assess the interaction between DUXAP8 and
Table I. Association between DUXAP8 expression and clinicopathological factors in patients with hepatocellular carcinoma.

\begin{tabular}{|c|c|c|c|c|}
\hline \multirow[b]{2}{*}{ Characteristic } & \multirow[b]{2}{*}{$\mathrm{n}$} & \multicolumn{2}{|c|}{ DUXAP8 expression } & \multirow[b]{2}{*}{ P-value } \\
\hline & & High $(\mathrm{n}=19)$ & Low $(n=19)$ & \\
\hline Age (years) & & & & 0.505 \\
\hline$<50$ & 17 & 10 & 7 & \\
\hline$\geq 50$ & 21 & 9 & 12 & \\
\hline Sex & & & & 0.282 \\
\hline Male & 20 & 12 & 8 & \\
\hline Female & 18 & 7 & 11 & \\
\hline HbsAg & & & & 0.304 \\
\hline Negative & 14 & 9 & 5 & \\
\hline Positive & 24 & 10 & 14 & \\
\hline Cirrhosis & & & & 0.410 \\
\hline Absent & 16 & 9 & 7 & \\
\hline Present & 22 & 10 & 12 & \\
\hline Tumor size $(\mathrm{cm})$ & & & & $0.031^{\mathrm{a}}$ \\
\hline$<5$ & 17 & 6 & 11 & \\
\hline$\geq 5$ & 21 & 13 & 8 & \\
\hline TNM stage & & & & $0.012^{\mathrm{a}}$ \\
\hline $\mathrm{I}, \mathrm{II}$ & 18 & 5 & 13 & \\
\hline III, IV & 20 & 14 & 6 & \\
\hline Metastasis & & & & $0.026^{\mathrm{a}}$ \\
\hline Yes & 15 & 5 & 10 & \\
\hline No & 23 & 14 & 9 & \\
\hline
\end{tabular}

${ }^{\mathrm{a}} \mathrm{P}<0.05$. DUXAP8, double homeobox A pseudogene 8 .

miR-9-3p, SK-HEP-1 and Huh-7 cells were co-transfected with DUXAP8-WT or DUXAP8-MUT reporter vector and miR-NC or miR-9-3p mimics, and the subsequent luciferase activities were measured. The results suggested that miR-9-3p overexpression significantly decreased the luciferase activity of the DUXAP8-WT reporter compared with miR-NC, but miR-9-3p overexpression had no significant effect on the luciferase activity of the DUXAP8-MUT reporter compared with miR-NC (Fig. 3E and F). Moreover, in accordance with the bioinformatics analysis and luciferase assay, the RIP assay results suggested that DUXAP8 and miR-9-3p were significantly enriched in Ago2 pellets of SK-HEP-1 and Huh-7 cell extracts compared with the IgG control group (Fig. 3G and $\mathrm{H}$ ). Meanwhile, the transfection efficiencies of miR-9-3p mimics and DUXAP8 overexpression were detected (Fig. S1). Moreover, miR-9-3p expression was significantly decreased in HCC tumor tissues and cell lines compared with adjacent healthy tissues and a human liver cell line, respectively (Fig. 3A and B). miR-9-3p expression levels were inversely correlated with DUXAP8 expression levels in HCC tumor tissues (Fig. 3C). Subsequently, miR-9-3p expression levels were detected in DUXAP8-transfected and si-DUXAP8-transfected SK-HEP-1 and Huh-7 cells. The RT-qPCR results suggested that DUXAP8 overexpression significantly downregulated miR-9-3p expression compared 
A

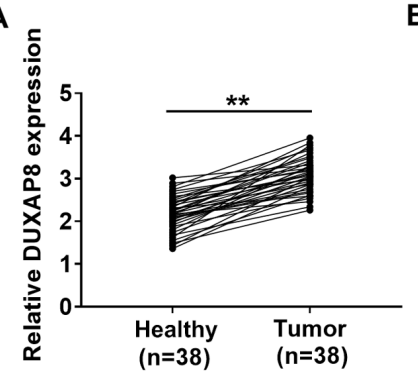

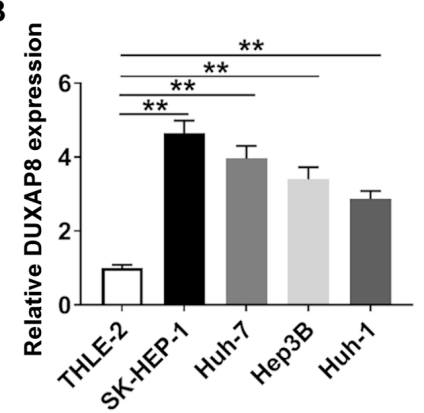

C

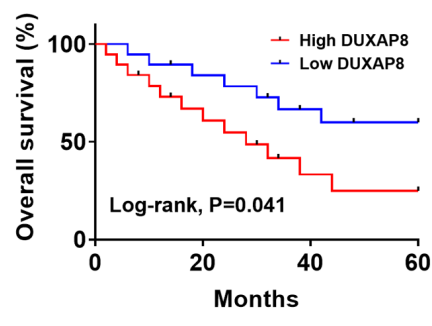

Figure 1. Expression patterns of DUXAP8 in HCC tissues and cells. (A) DUXAP8 expression levels in 38 paired HCC tumor and adjacent healthy tissues. (B) DUXAP8 expression levels in HCC cell lines (SK-HEP-1, Huh-7, Hep38 and Huh-1) and a normal human liver cell line (THLE-2). (C) Kaplan-Meier overall survival curves of patients with HCC based on the median expression of DUXAP8 expression levels. $\mathrm{n}=3$. ${ }^{* *} \mathrm{P}<0.01$. DUXAP8, double homeobox A pseudogene 8; HCC, hepatocellular carcinoma.

A

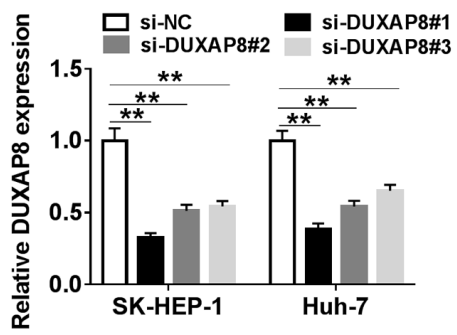

D
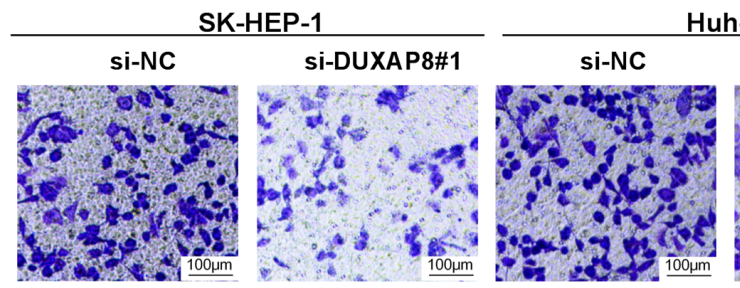

Huh-7

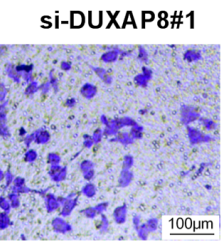

$\mathbf{E}$

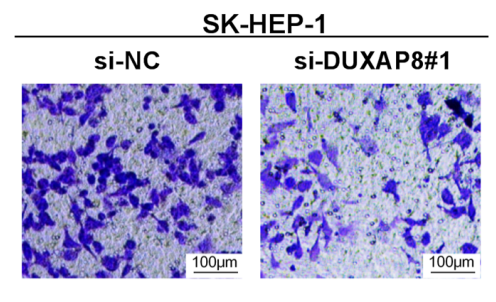

$\mathbf{F}$

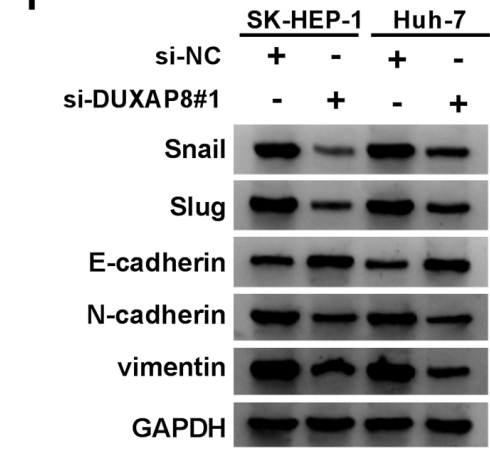

B

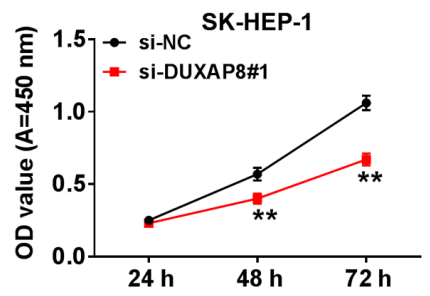

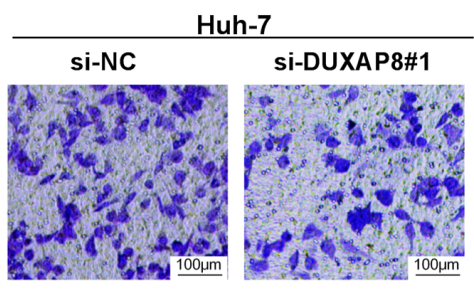

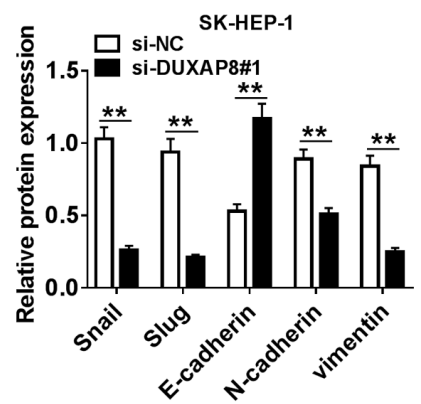

C

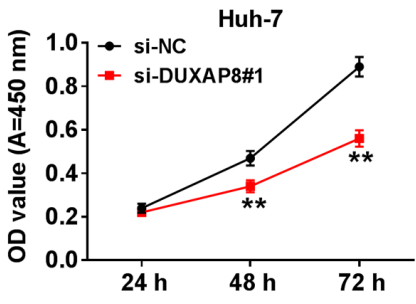

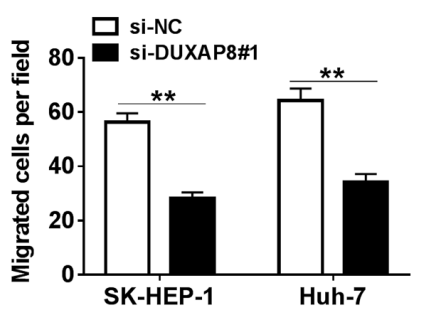
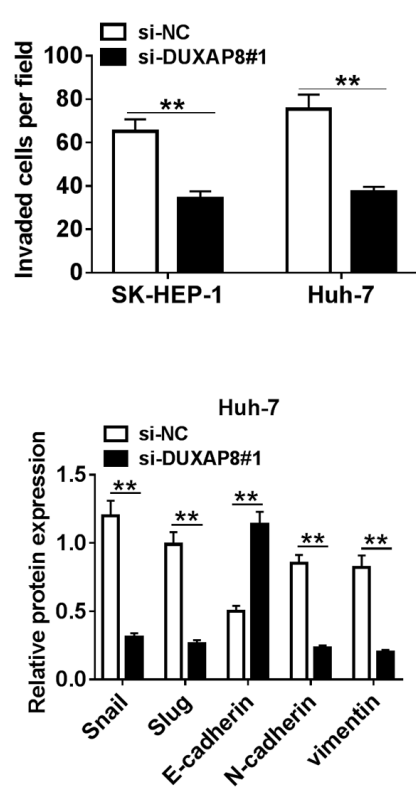

Figure 2. DUXAP8 induces HCC cell proliferation, migration, invasion and EMT. (A) Knockdown efficiency of si-DUXAP8 in SK-HEP-1 and Huh-7 cells. Cell proliferation in si-DUXAP8-transfected (B) SK-HEP-1 and (C) Huh-7 cells. Cell (D) migration and (E) invasion in si-DUXAP8-transfected SK-HEP-1 and Huh-7 cells. (F) Expression levels of EMT-related proteins (Snail, Slug, E-cadherin, N-cadherin and vimentin) were detected via western blotting in si-DUXAP8-transfected SK-HEP-1 and Huh-7 cells. $\mathrm{n}=3$. ${ }^{* *} \mathrm{P}<0.01$. DUXAP8, double homeobox A pseudogene 8; HCC, hepatocellular carcinoma; EMT, epithelial-mesenchymal transition; si, small interfering RNA; NC, negative control; OD, optical density. 
A

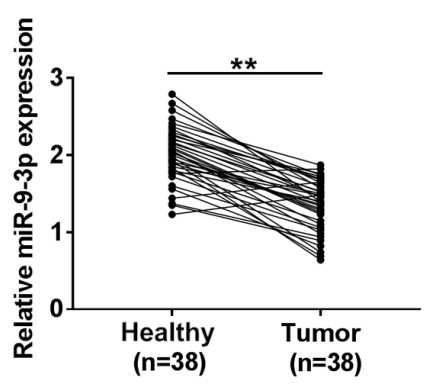

D

Target site: chr22:16151502-16151523

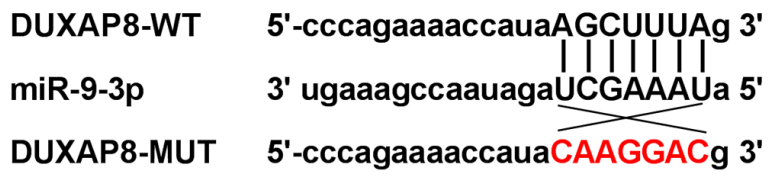

$\mathbf{F}$

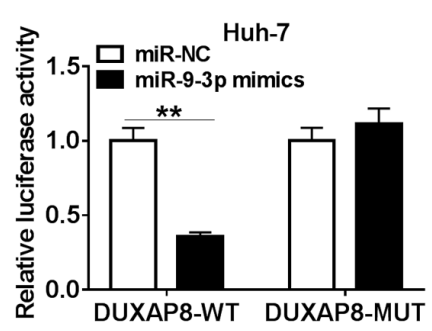

I

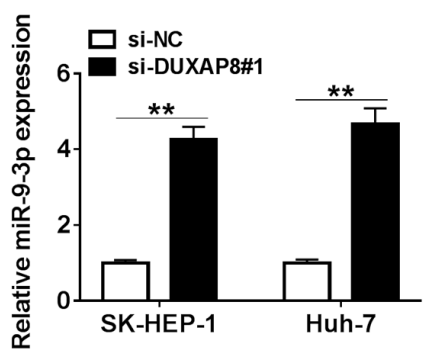

B

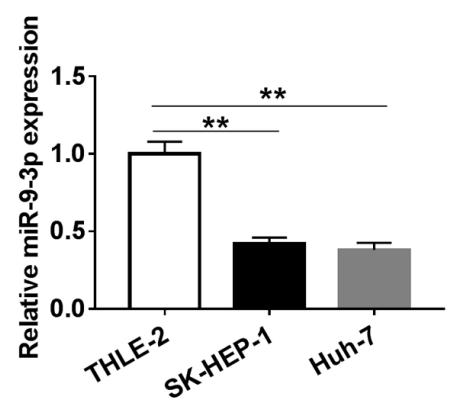

C

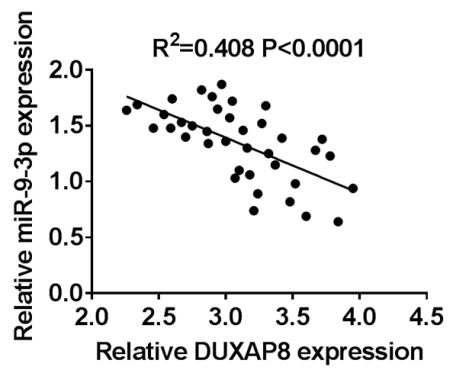

$\mathbf{E}$

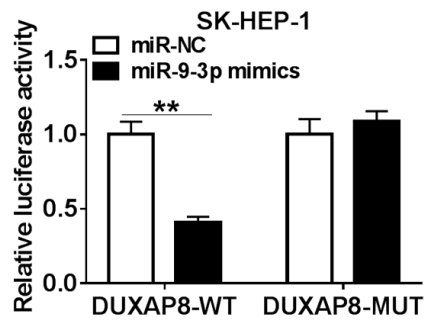

H
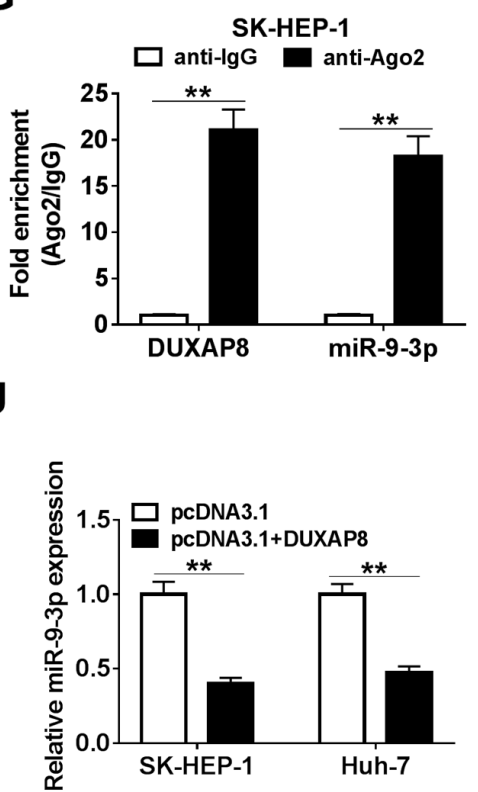

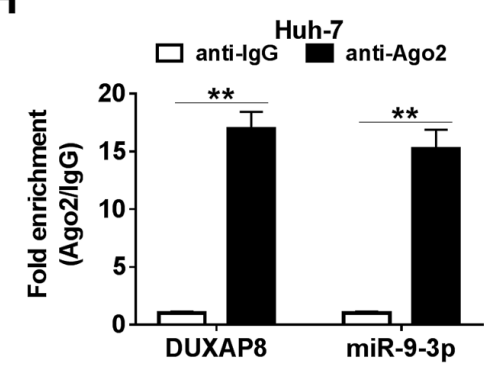

Figure 3. miR-9-3p is a direct target of DUXAP8 in HCC cells. (A) Expression of miR-9-3p in 38 paired HCC tumor and adjacent healthy tissues. (B) Expression of miR-9-3p in HCC cell lines (SK-HEP-1, Huh-7, Hep38 and Huh-1) and a normal human liver cell line (THLE-2). (C) Correlation between DUXAP8 and miR-9-3p in HCC tissues. (D) Binding sites between DUXAP8 and miR-9-3p. The relative luciferase activity in (E) SK-HEP-1 and (F) Huh-7 cells co-transfected with DUXAP8-WT or DUXAP8-MUT vectors and miR-NC or miR-9-3p mimics. An RNA immunoprecipitation assay was conducted in (G) SK-HEP-1 and (H) Huh-7 cell extracts to examine whether miR-9-3p endogenously associated with DUXAP8. The effects of DUXAP8 (I) knockdown and $(\mathrm{J})$ overexpression on miR-9-3p expression were detected via reverse transcription-quantitative PCR in SK-HEP-1 and Huh-7 cells. $\mathrm{n}=3$. ${ }^{* * *} \mathrm{P}<0.01$. miR, microRNA; DUXAP8, double homeobox A pseudogene 8; HCC, hepatocellular carcinoma; WT, wild-type; MUT, mutant; NC, negative control; si, small interfering RNA; Ago2, Argonaute2.

with pcDNA3.1, whereas DUXAP8 knockdown significantly upregulated miR-9-3p expression levels compared with si-NC (Fig. 3I and J), suggesting that manipulation of DUXAP8 expression altered the expression of miR-9-3p. The results suggested that DUXAP8 interacted with miR-9-3p to repress its expression.

DUXAP8 facilitates HCC progression by targeting miR-9-3p. To further investigate the mechanism underlying DUXAP8 in $\mathrm{HCC}$ progression, rescue experiments were performed by transfecting SK-HEP-1 and Huh-7 cells with si-NC, si-DUXAP8, si-DUXAP8 + anti-miR-NC or si-DUXAP8 + miR-9-3p inhibitor. The RT-qPCR results indicated that DUXAP8 knockdown significantly increased miR-9-3p expression levels compared with si-NC, which were significantly reversed by co-transfection with miR-9-3p inhibitor in SK-HEP-1 and Huh-7 cells (Fig. 4A). The transfection efficiency of miR-9-3p inhibitor was examined (Fig. S1). Furthermore, compared with si-NC, DUXAP8 knockdown significantly inhibited SK-HEP-1 and Huh-7 cell proliferation, migration 
A

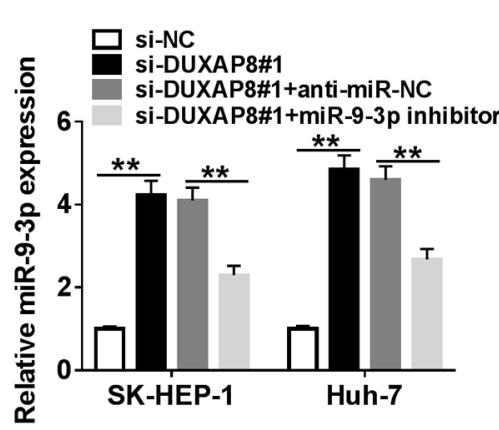

D

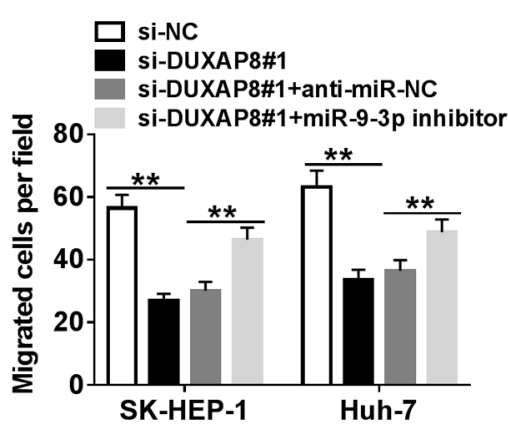

G

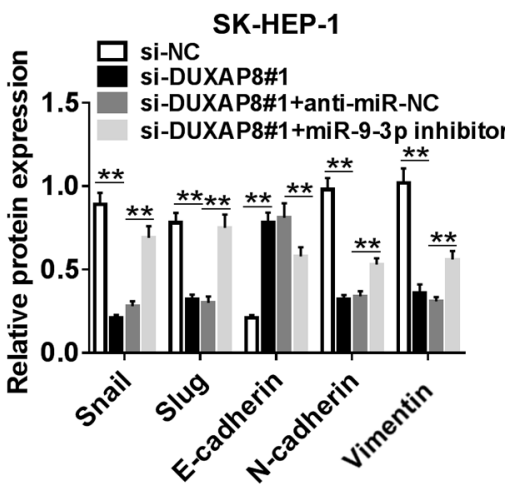

B

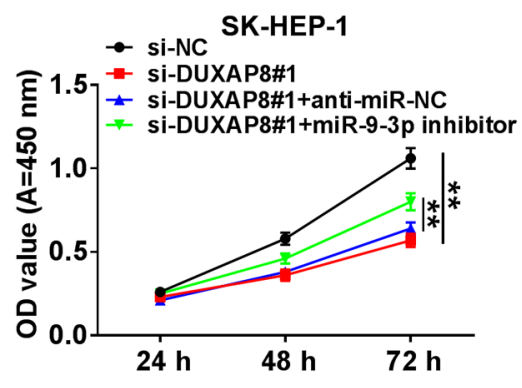

E

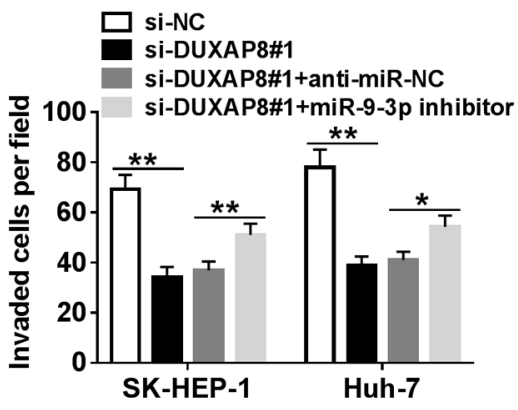

H

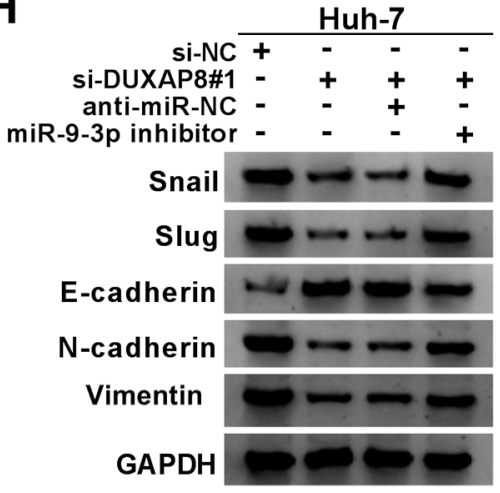

C
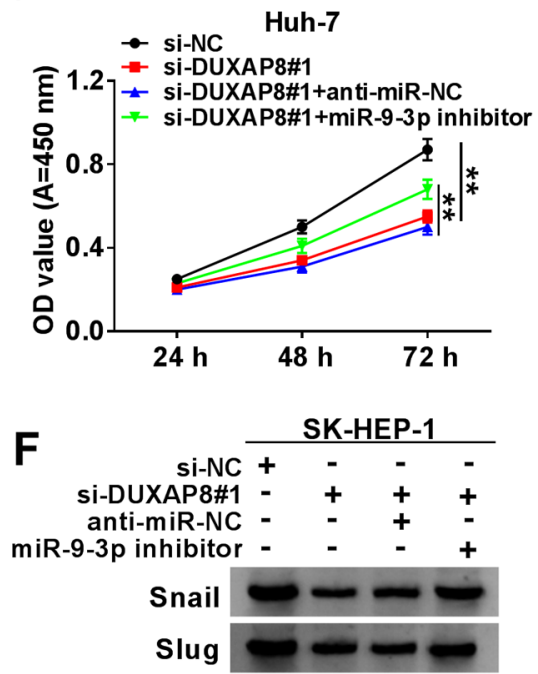

E-cadherin

N-cadherin

Vimentin

GAPDH
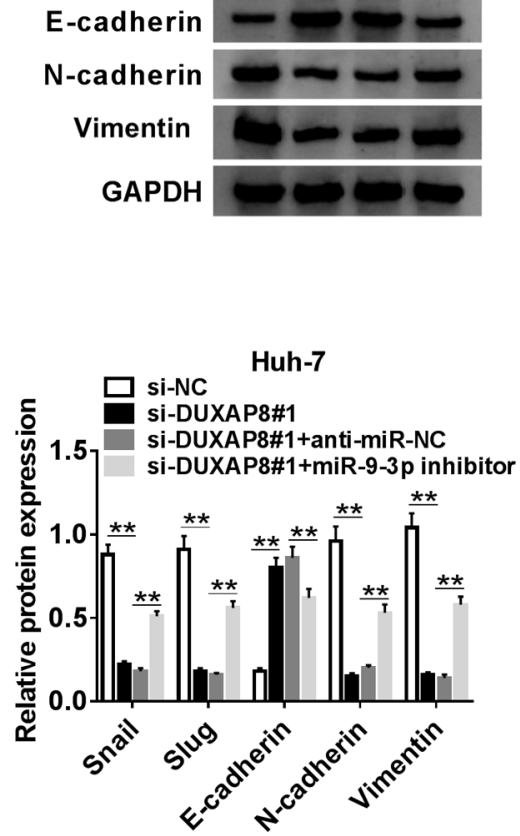

Figure 4. DUXAP8 facilitates HCC progression by targeting miR-9-3p. (A) miR-9-3p expression levels in SK-HEP-1 and Huh-7 cells transfected with si-NC, si-DUXAP8, si-DUXAP8 + anti-miR-NC or si-DUXAP8 + miR-9-3p inhibitor. Cell proliferation in (B) SK-HEP-1 and (C) Huh-7 cells. Cell (D) migration and (E) invasion in transfected SK-HEP-1 and Huh-7 cells. EMT-related protein expression levels were (F) determined by western blotting and (G) semi-quantified in SK-HEP-1 cells. EMT-related protein expression levels were $(\mathrm{H})$ determined by western blotting and semi-quantified in Huh-7 cells. $\mathrm{n}=3$. ${ }^{*} \mathrm{P}<0.05 ;{ }^{* *} \mathrm{P}<0.01$. DUXAP8, double homeobox A pseudogene 8; HCC, hepatocellular carcinoma; miR, microRNA; si, small interfering RNA; NC, negative control.

and invasion, and miR-9-3p knockdown reversed DUXAP8 knockdown-mediated effects (Figs. 4B-E, S2A and S2B). The western blotting results suggested that miR-9-3p inhibition significantly decreased si-DUXAP8-induced increases in E-cadherin protein expression levels, and decreases in Snail, Slug, N-cadherin and vimentin protein expression levels in SK-HEP-1 and Huh-7 cells (Fig. 4F-I). Collectively, the results suggested that DUXAP8 may exert its carcinogenic effects partially by regulating miR-9-3p.

IGFIR is a target of miR-9-3p in HCC cells. Previous reports have demonstrated that miRNAs may exert their function by interacting with mRNAs (37-39). Therefore, TargetScan was used to analyze the potential downstream target of miR-9-3p. IGF1R was predicted to possess binding sites with miR-9-3p
(Fig. 5A). To validate the bioinformatics prediction, SK-HEP-1 and Huh-7 cells were co-transfected with IGF1R-WT or IGF1R-MUT reporter plasmids and miR-NC or miR-9-3p mimics. The luciferase assay suggested that compared with miR-NC, miR-9-3p overexpression significantly inhibited the luciferase activity of the IGF1R-WT reporter, but had no significant effect on the luciferase activity of the IGF1R-MUT reporter (Fig. 5B and C). Subsequently, IGF1R expression in miR-9-3p mimics- or miR-9-3p inhibitor-transfected SK-HEP-1 and Huh-7 cells was detected. The RT-qPCR and western blotting results suggested that miR-9-3p expression altered the expression of IGF1R, as evidenced by miR-9-3p overexpression significantly decreasing IGF1R expression levels compared with miR-NC, and miR-9-3p knockdown significantly increasing IGF1R expression levels compared 
A

Binding Site:chr15:99506460-99506488

IGF1R 3'UTR-WT 5'-CCUGUUUACUAGCUAGCUUUAC-3'

miR-9-3p 3' UGAAAGCCAAUAGAUCGAAAUA 5'

IGF1R 3' UTR-MUT 5'-CCUGUUUACUAGAGGAACCGCA-3'

C

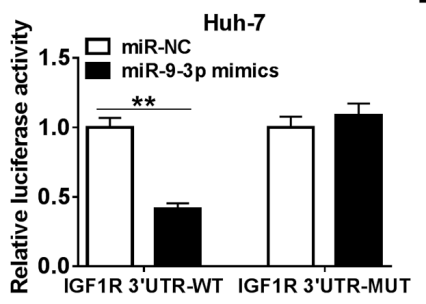

$\mathbf{F}$

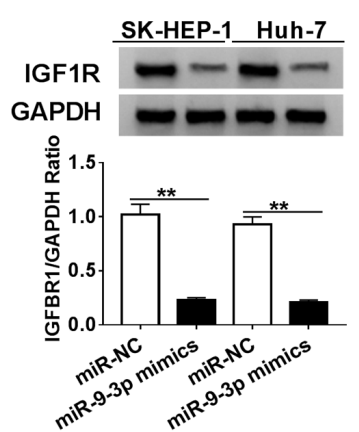

I

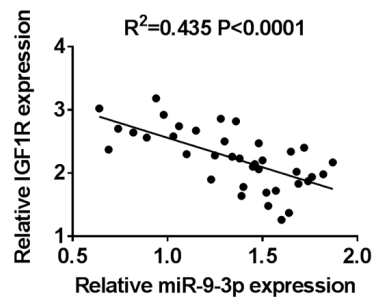

B

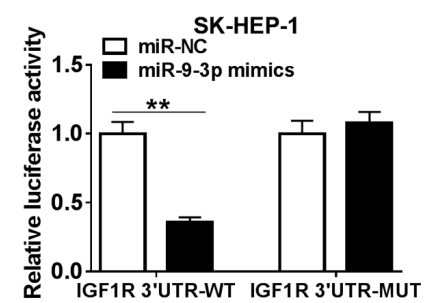

E

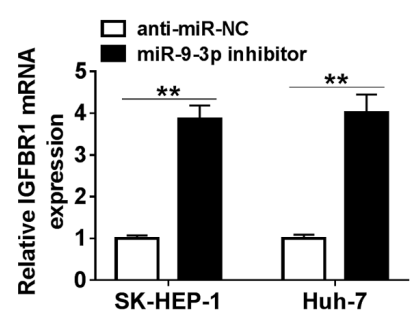

H

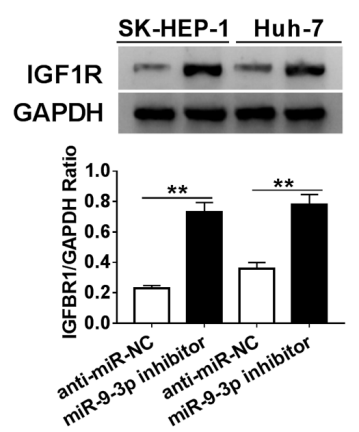

Figure 5. IGF1R is a target of miR-9-3p. (A) Putative binding sequences between miR-9-3p and IGF1R. The relative luciferase activity of (B) SK-HEP-1 and (C) Huh-7 cells co-transfected with IGF1R 3'UTR-WT or IGF1R 3'UTR-MUT vectors and miR-NC or miR-9-3p mimics. The effects of miR-9-3p (D) overexpression and (E) knockdown on IGF1R expression in SK-HEP-1 and Huh-7 cells. IGF1R expression levels in SK-HEP-1 and Huh-7 cells transfected with (F) miR-NC, miR-9-3p mimics, (G) anti-miR-NC or miR-9-3p inhibitor. (H) Expression of IGF1R in 38 paired HCC tumor and adjacent healthy tissues. (I) Correlation between IGF1R and miR-9-3p expression in HCC tumor tissues. $n=3{ }^{* *} \mathrm{P}<0.01$. IGF1R, insulin-like growth factor 1 receptor; miR, microRNA; UTR, untranslated region; WT, wild-type; MUT, mutant; NC, negative control; HCC, hepatocellular carcinoma.

with anti-miR-NC in SK-HEP-1 and Huh-7 cells (Fig. 5D-G). Moreover, IGF1R expression was significantly upregulated and negatively correlated with miR-9-3p expression in HCC tumor tissues (Fig. 5H and I). Collectively, the results suggested that IGF1R was a target of miR-9-3p in HCC cells.

miR-9-3p suppresses HCC progression by targeting IGFIR. To further investigate whether miR-9-3p could exert its function via targeting in HCC cells, SK-HEP-1 and Huh-7 cells were transfected with miR-NC, miR-9-3p mimics, miR-9-3p mimics + pcDNA3.1 or miR-9-3p mimics + pcDNA3.1-IGF1R The RT-qPCR and western blotting results indicated that miR-9-3p overexpression significantly decreased the expression of IGF1R in SK-HEP-1 and Huh-7 cells compared with miR-NC, which was reversed by co-transfection with
pcDNA3.1-IGF1R (Fig. 6A-C). In addition, according to the analysis performed using the Pan-Cancer platform of StarBase, the expression correlation of DUXAP8, IGF1R and miR-9-3p in LIHC samples was assessed. The expression of miR-9-3p was positively correlated with that of DUXAP8 or IGF1R, and DUXAP8 expression was positively correlated with that of IGF1R (Fig. S3). Simultaneously, the transfection efficiency of IGF1R overexpression was measured (Fig. S1). Compared with miR-NC, miR-9-3p overexpression significantly inhibited SK-HEP-1 and Huh-7 cell proliferation, migration and invasion, which was reversed by IGF1R overexpression (Figs. 6D-G, S2C and S2D). In addition, compared with miR-NC, miR-9-3p overexpression significantly increased E-cadherin protein expression levels, and decreased Snail, Slug, N-cadherin and vimentin protein 
A

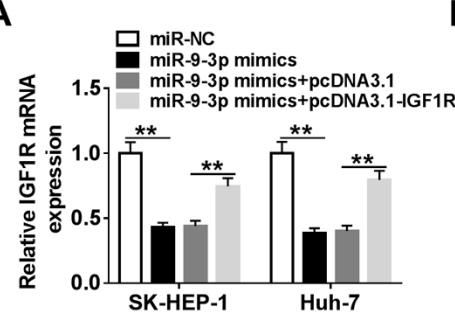

E

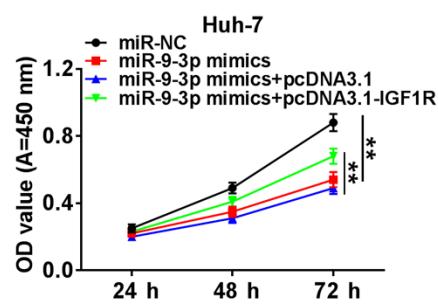

I

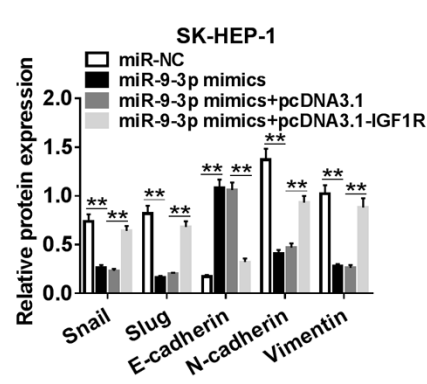

B

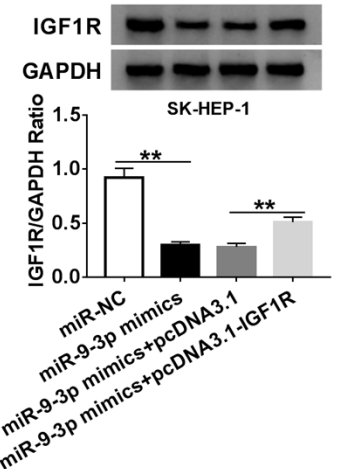

$F$
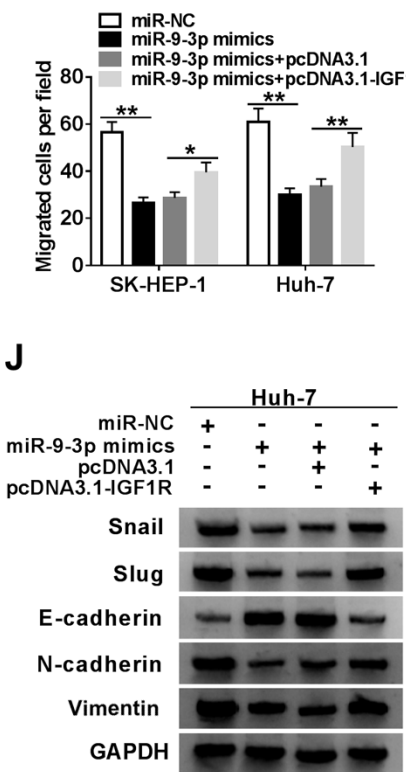

C

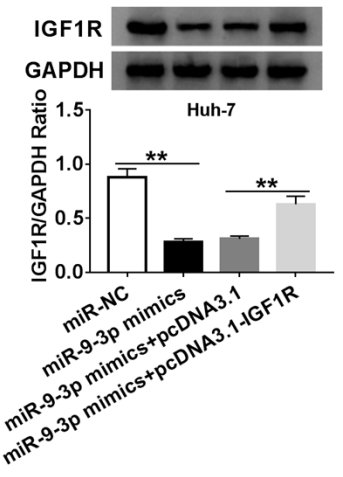

G

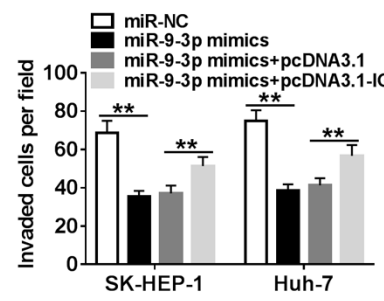

K

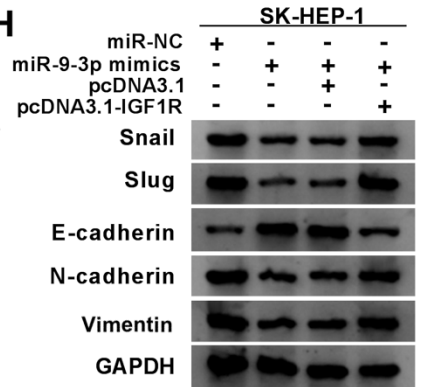

Figure 6. miR-9-3p suppresses HCC progression by targeting IGF1R. (A) IGF1R expression in SK-HEP-1 and Huh-7 cells. IGF1R protein expression levels in (B) SK-HEP-1 and (C) Huh-7 cells. Cell proliferation in (D) SK-HEP-1 and (E) Huh-7 cells transfected with miR-NC, miR-9-3p mimics, miR-9-3p mimics + pcDNA3.1 or miR-9-3p mimics + IGF1R. Cell (F) migration and (G) invasion in SK-HEP-1 and Huh-7 cells transfected with miR-NC, miR-9-3p mimics, miR-9-3p mimics + pcDNA3.1 or miR-9-3p mimics + IGF1R. (H and I) EMT-related protein expression levels were (H) determined by western blotting and (I) semi-quantified in SK-HEP-1 cells. EMT-related protein expression levels were (J) determined by western blotting and (K) semi-quantified in Huh-7 cells. $\mathrm{n}=3$. ${ }^{*} \mathrm{P}<0.05 ;{ }^{* *} \mathrm{P}<0.01$. miR, microRNA; HCC, hepatocellular carcinoma; IGF1R, insulin-like growth factor 1 receptor; NC, negative control; EMT, epithelial-mesenchymal transition; OD, optical density.

expression levels, which were reversed by co-transfection with pcDNA3.1-IGF1R (Fig. 6H and I), indicating that IGF1R abolished miR-9-3p-mediated inhibition of SK-HEP-1 and Huh-7 cell EMT. The results indicated that miR-9-3p repressed HCC development by modulating IGF1R.

Verification of the DUXAP8/miR-9-3p/IGFIR regulatory axis in HCC cells. According to the aforementioned results, it was hypothesized that DUXAP8 could exert its carcinogenic effects via the DUXAP8/miR-9-3p/IGF1R regulatory signaling pathway. Furthermore, DUXAP8 expression was positively correlated with IGF1R expression in HCC tumor tissues (Fig. 7A). Subsequently, whether DUXAP8 could regulate the expression of IGF1R via miR-9-3p was investigated. The RT-qPCR and western blotting results suggested that DUXAP8 knockdown reduced the expression of IGF1R compared with si-NC, and co-transfection with miR-9-3p inhibitor reversed si-DUXAP8-mediated inhibitory effects on IGF1R expression in SK-HEP-1 and Huh-7 cells (Fig. 7B-D).
Collectively, the results suggested that DUXAP8 may serve as a molecular sponge to sequester miR-9-3p from IGF1R in HCC cells.

\section{Discussion}

Numerous studies have indicated that lncRNAs are aberrantly expressed and serve as diagnostic and prognostic biomarkers in HCC (40-42). LncRNA DUXAP8, as an oncogene, is dysregulated in multiple tumors, such as bladder cancer (13), non-small-cell lung cancer (43) and ovarian cancer (44). For instance, Xu et al (45) demonstrated that DUXAP8 was overexpressed in esophageal squamous cell cancer tissues, and DUXAP8 knockdown blocked proliferation, invasion and colony formation in vitro. Lin et al (46) reported that DUXAP8 downregulation inhibited proliferation by binding to phosphatase and tensin homolog in bladder cancer. Nevertheless, the exact function and mechanisms underlying DUXAP8 in HCC progression are not completely understood. 
A

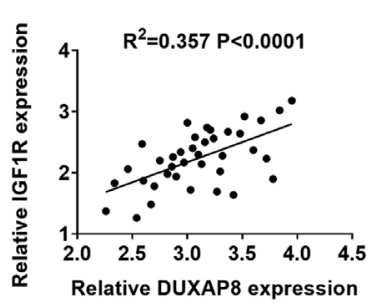

C

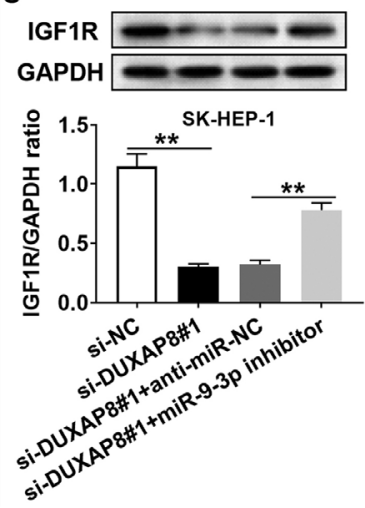

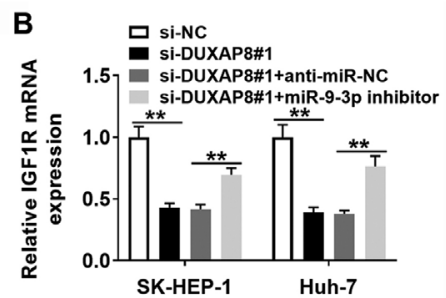

D

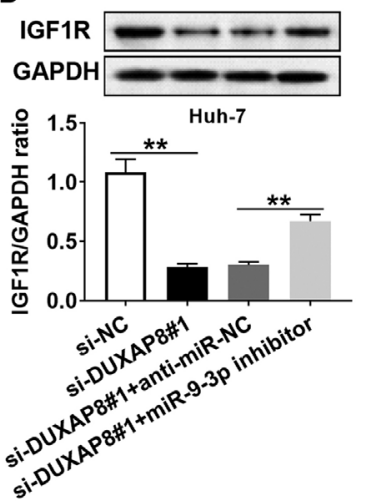

Figure 7. DUXAP8 regulates IGF1R expression by sponging miR-9-3p. (A) Correlation between DUXAP8 and IGF1R expression in hepatocellular carcinoma tumor tissues. (B) IGF1R expression in SK-HEP-1 and Huh-7 cells transfected with si-NC, si-DUXAP8, si-DUXAP8 + anti-miR-NC and si-DUXAP8 + miR-9-3p inhibitor. IGF1R protein expression levels in transfected (C) SK-HEP-1 and (D) Huh-7 cells. $n=3 .{ }^{* *} \mathrm{P}<0.01$. DUXAP8, double homeobox A pseudogene 8; IGF1R, insulin-like growth factor 1 receptor; miR, microRNA; si, small interfering RNA; NC, negative control.

miR-9-3p has been confirmed as a tumor suppressor in a variety of different types of cancer, such as glioma (47), nasopharyngeal carcinoma (48) and bladder cancer (49). Of interest, in HCC cells, Yang et al (50) demonstrated that miR-9-3p overexpression reduced the migration and invasion rates of HCC in vitro. Tang et al (51) demonstrated that miR-9-3p, a functional biomarker, inhibited cell proliferation by suppressing human fibroblast growth factor-5 expression in HCC.

In the present study, the expression level of DUXAP8 in HCC tumor tissues and cells was measured. The results indicated that DUXAP8 expression was significantly upregulated in HCC tumor tissues and cells compared with adjacent healthy tissues and a normal liver cell line, respectively. Moreover, the clinicopathologic factors and the prognostic value of HCC were assessed. The results indicated that DUXAP8 expression was significantly associated with tumor size, TNM stage and metastasis, and patients with high DUXAP8 expression displayed a worse overall survival rate compared with patients with low DUXAP8 expression, suggesting that DUXAP8 served a vital role in the development and progression of $\mathrm{HCC}$.

The effect of DUXAP8 on HCC cell proliferation, migration, invasion and EMT was evaluated. Functional analyses indicated that DUXAP8 enhanced HCC cell proliferation, migration, invasion and EMT, indicating that DUXAP8 performed as an oncogene in HCC progression. Subsequently, the potential molecular mechanisms underlying DUXAP8 in $\mathrm{HCC}$ development were investigated. In recent decades,

several studies have identified that lncRNA could serve as a miRNA sponge to interact with miRNAs $(52,53)$. Therefore, StarBase was used to predict the underlying target miRNAs of DUXAP8. As a result, miR-9-3p was found to possess binding sites of DUXAP8, which was further indicated by the luciferase reporter assay. Moreover, in the present study, miR-9-3p expression was downregulated and inversely correlated with DUXAP8 expression in HCC tumor tissues and cells. Consequently, whether the effect of DUXAP8 on HCC progression was mediated via the regulation of miR-9-3p was investigated. In the present study, the results verified that silencing DUXAP8 inhibited HCC cell proliferation, migration, invasion and EMT, whereas miR-9-3p knockdown attenuated the effects of DUXAP8 on HCC cells. Therefore, to the best of our knowledge, the present study indicated for the first time that DUXAP8 served as an oncogenic factor in HCC development potentially via targeting the expression of miR-9-3p.

Increasing studies have focused onlncRNA-miRNA-mRNA regulatory axes, suggesting that lncRNAs serve as competing endogenous RNAs (ceRNAs) to sequester miRNAs away from target mRNAs, leading to the upregulation of mRNA expression (54-56). Therefore, IGF1R as the target gene of miR-9-3p in HCC cells was identified using TargetScan software and verified by performing luciferase reporter assays. As a subunit of the transmembrane receptor family, IGF1R participates in various biological processes, including embryogenesis, tissue repair and metastatic diffusion of tumor cells (57). A previous study also indicated that IGF1R triggered HCC cell metastasis and proliferation (58). In the present study, compared with adjacent healthy tissues, IGF1R expression was upregulated and negatively correlated with miR-9-3p expression in HCC tumor tissues. Therefore, whether IGF1R was involved in mediating miR-9-3p expression in HCC development was investigated. The results indicated that, compared with miR-NC, miR-9-3p overexpression inhibited proliferation, migration, invasion and EMT and IGF1R overexpression partly reversed miR-9-3p-induced suppressive effects in HCC cells, indicating that miR-9-3p may inhibit tumor progression partially by targeting IGF1R.

In addition, to further assess whether DUXAP8 served as a ceRNA of miR-9-3p by interacting with IGF1R, the effect of DUXAP8 on IGF1R expression was investigated. In the present study, IGF1R expression was positively correlated with DUXAP8 expression in HCC tumor tissues. Compared with si-NC, DUXAP8 knockdown decreased IGF1R expression, which was reversed by co-transfection with miR-9-3p inhibitor in HCC cells. In addition, the association among DUXAP8, IGF1R and miR-9-3p was also analyzed using the Pan-Cancer platform of StarBase, and indicated that miR-9-3p was positively correlated with DUXAP8 or IGF1R, and DUXAP8 expression was positively correlated with that of IGF1R. The aforementioned results differed from the other results of the present study, which might be due to the difference between high-throughput predicted data and actual gene expression. In the present study, RT-qPCR was performed to detect the expression of each gene in patient samples, and the obtained data should be more accurate.

In conclusion, the present study suggested that DUXAP8 served as a miR-9-3p sponge to upregulate IGF1R expression, 
thereby enhancing the development of HCC. The present study also indicated that targeting the DUXAP8/miR-9-3p/IGF1R regulatory axis may serve as a promising therapeutic approach for HCC.

\section{Acknowledgements}

Not applicable.

\section{Funding}

No funding was received.

\section{Availability of data and materials}

The datasets used and/or analyzed during the current study are available from the corresponding author on reasonable request.

\section{Authors' contributions}

QG and BY designed the study. XZ and TY performed the experiments. JL and WX analyzed and interpreted the data. WX drafted the manuscript. All authors read and approved the final manuscript.

\section{Ethics approval and consent to participate}

The present study was approved by People's Hospital of Dongying Ethics Committee (Dongying, China). Written informed consent was obtained from all participants.

\section{Patient consent for publication}

Not applicable.

\section{Competing interests}

The authors declare that they have no competing interests.

\section{References}

1. Siegel RL, Miller KD and Jemal A: Cancer statistics, 2017. CA Cancer J Clin 67: 7-30, 2017.

2. Bray F, Ferlay J, Soerjomataram I, Siegel RL, Torre LA and Jemal A: Global cancer statistics 2018: GLOBOCAN estimates of incidence and mortality worldwide for 36 cancers in 185 countries. CA Cancer J Clin 68: 394-424, 2018.

3. Erstad DJ and Tanabe KK: Prognostic and therapeutic implications of microvascular invasion in hepatocellular carcinoma Ann Surg Oncol 26: 1474-1493, 2019.

4. Mercer TR, Dinger ME and Mattick JS: Long Non-coding RNAs: Insights into functions. Nat Rev Genet 10: 155-159, 2009

5. Marchese FP, Raimondi I and Huarte M: The multidimensional mechanisms of long noncoding RNA function. Genome Biol 18 206, 2017.

6. Kong L and Zhang C: LncRNA DLX6-AS1 aggravates the development of ovarian cancer via modulating FHL2 by sponging miR-195-5p. Cancer Cell Int 20: 370, 2020.

7. Yang Q, Wan Q, Zhang L, Li Y, Zhang P, Li D, Feng C, Yi F, Zhang L, Ding X, et al: Analysis of LncRNA expression in cell differentiation. RNA Biol 15: 413-422, 2018

8. Wu Y and Qian Z: Long non-coding RNAs (lncRNAs) and microRNAs regulatory pathways in the tumorigenesis and pathogenesis of glioma. Discov Med 28: 129-138, 2019.
9. Yang Y, Chen L, Gu J, Zhang H, Yuan J, Lian Q, Lv G, Wang S, $\mathrm{Wu}$ Y, Yang YT, et al: Recurrently deregulated lncRNAs in hepatocellular carcinoma. Nat Commun 8: 14421, 2017.

10. Li B, Mao R, Liu C, Zhang W, Tang Y and Guo Z: LncRNA FAL1 promotes cell proliferation and migration by acting as a CeRNA of miR-1236 in hepatocellular carcinoma cells. Life Sci 197: 122-129, 2018.

11. Ma J, Li T and Han X: Knockdown of LncRNA ANRIL suppresses cell proliferation, metastasis, and invasion via regulating miR-122-5p expression in hepatocellular carcinoma. J Cancer Res Clin Oncol 144: 205-214, 2018.

12. Zhao X, Hao S, Wang M, Xing D and Wang C: Knockdown of pseudogene DUXAP8 expression in glioma suppresses tumor cell proliferation. Oncol Lett 17: 3511-3516, 2019.

13. Jiang B, Hailong S, Yuan J, Zhao H, Xia W, Zha Z, Bin W and Liu Z: Identification of oncogenic long noncoding RNA SNHG12 and DUXAP8 in human bladder cancer through a comprehensive profiling analysis. Biomed Pharmacother 108: 500-507, 2018

14. Huang T, Wang X, Yang X, Ji J, Wang Q, Yue X and Dong Z: Long non-coding RNA DUXAP8 enhances renal cell carcinoma progression via downregulating miR-126. Med Sci Monit 24: 7340-7347, 2018

15. Sun M, Nie FQ, Zang C, Wang Y, Hou J, Wei C, Li W, He X and Lu KH: The pseudogene DUXAP8 promotes non-small-cell lung cancer cell proliferation and invasion by epigenetically silencing EGR1 and RHOB. Mol Ther 25: 739-751, 2017.

16. Afonso-Grunz F and Muller S: Principles of miRNA-mRNA interactions: Beyond sequence complementarity. Cell Mol Life Sci 72: 3127-3141, 2015.

17. Zha Z, Jia F, Hu P, Mai E and Lei T: MicroRNA-574-3p inhibits the malignant behavior of liver cancer cells by targeting ADAM28. Oncol Lett 20: 3015-3023, 2020

18. Liu CH, Jing XN, Liu XL, Qin SY, Liu MW and Hou CH: Tumor-suppressor miRNA-27b-5p regulates the growth and metastatic behaviors of ovarian carcinoma cells by targeting CXCL1. J Ovarian Res 13: 92, 2020.

19. Fang F, Chang RM, Yu L, Lei X, Xiao S, Yang H and Yang LY: MicroRNA-188-5p suppresses tumor cell proliferation and metastasis by directly targeting FGF5 in hepatocellular carcinoma. J Hepatol 63: 874-885, 2015.

20. Chen Y, Zhao ZX, Huang F, Yuan XW, Deng L and Tang D: MicroRNA-1271 functions as a potential tumor suppressor in hepatitis B virus-associated hepatocellular carcinoma through the AMPK signaling pathway by binding to CCNA1. J Cell Physiol 234: 3555-3569, 2019.

21. Higashi T, Hayashi H, Ishimoto T, Takeyama H, Kaida T, Arima K, Taki K, Sakamoto K, Kuroki H, Okabe H, et al: MiR-9-3p plays a tumour-suppressor role by targeting TAZ (WWTR1) in hepatocellular carcinoma cells. Br J Cancer 113: 252-258, 2015.

22. Erickson KE and Rukhlenko OS: Modeling cell line-specific recruitment of signaling proteins to the insulin-like growth factor 1 receptor. PLoS Comput Biol 15: e1006706, 2019.

23. Lin JF, Tsai TF, Lin YC, Chen HE, Chou KY and Hwang TI: Benzyl isothiocyanate suppresses IGF1R, FGFR3 and mTOR expression by upregulation of miR-99a-5p in human bladder cancer cells. Int J Oncol 54: 2106-2116, 2019.

24. Zhang Y, Huang S, Guo Y and Li L: MiR-1294 confers cisplatin resistance in ovarian Cancer cells by targeting IGF1R. Biomed Pharmacother 106: 1357-1363, 2018.

25. Khalil A and Jameson MJ: Downregulation of igf1r expression inhibits growth and enhances cisplatin sensitivity of head and neck squamous cell carcinoma cells in vitro. Horm Cancer 10: $11-23,2019$.

26. Liu W, Kang L, Han J, Wang Y, Shen C, Yan Z, Tai Y and Zhao C: MiR-342-3p suppresses hepatocellular carcinoma proliferation through inhibition of IGF-1R-mediated Warburg effect. Onco Targets Ther 11: 1643-1653, 2018.

27. Ren L, Yao Y, Wang Y and Wang S: MiR-505 suppressed the growth of hepatocellular carcinoma cells via targeting IGF-1R. Biosci Rep 39: BSR20182442, 2019.

28. Ye Y, Zhuang J, Wang G, He S, Zhang S, Wang G, Ni J, Wang J and Xia W: MicroRNA-495 suppresses cell proliferation and invasion of hepatocellular carcinoma by directly targeting insulin-like growth factor receptor-1. Exp Ther Med 15: 1150-1158, 2018

29. Liu MD, Wu H, Wang S, Pang P, Jin S, Sun CF and Liu FY: MiR-1275 promotes cell migration, invasion and proliferation in squamous cell carcinoma of head and neck via up-regulating IGF-1R and CCR7. Gene 646: 1-7, 2018 
30. Zhou Y, Zhang Z, Wang N, Chen J, Zhang X, Guo M, John Zhong L and Wang Q: Suppressor of cytokine signalling-2 limits IGF1R-mediated regulation of epithelial-mesenchymal transition in lung adenocarcinoma. Cell Death Dis 9: 429, 2018.

31. Livak KJ and Schmittgen TD: Analysis of relative gene expression data using real-time quantitative PCR and the 2(-Delta Delta C(T)) method. Methods 25: 402-408, 2001.

32. Li JH, Liu S, Zhou H, Qu LH and Yang JH: StarBase v2.0: Decoding miRNA-ceRNA, miRNA-ncRNA and protein-RNA interaction networks from large-scale CLIP-Seq data. Nucleic Acids Res 42 (Database Issue): D92-D97, 2014.

33. Yang JH, Li JH, Shao P, Zhou H, Chen YQ and Qu LH: StarBase: A database for exploring microRNA-mRNA interaction maps from Argonaute CLIP-Seq and Degradome-Seq data. Nucleic Acids Res 39 (Database Issue): D202-D209, 2011.

34. Li Y: MIR31HG exhibits oncogenic property and acts as a sponge for miR-361-3p in cervical carcinoma. Biochem Biophys Res Commun 529: 890-897, 2020.

35. Sun F and Wu K: Long Noncoding RNA PVT1 promotes prostate cancer metastasis by increasing NOP2 expression via targeting tumor suppressor MicroRNAs. Onco Targets Ther 13: 6755-6765, 2020.

36. Shi X, Huo J, Gao X, Cai H and Zhu W: A newly identified lncRNA H1FX-AS1 targets DACT1 to inhibit cervical cancer via sponging miR-324-3p. Cancer Cell Int 20: 358, 2020.

37. Wu R, Zhao B, Ren X, Wu S, Liu M, Wang Z and Liu W: MiR-27a-3p targeting GSK3 $\beta$ promotes triple-negative breast cancer proliferation and migration through $\mathrm{Wnt} / \beta$-catenin pathway. Cancer Manag Res 12: 6241-6249, 2020.

38. Li G, Qi HW, Dong HG, Bai P, Sun M and Liu HY: Targeting deubiquitinating enzyme USP26 by microRNA-203 regulates Snaill's pro-metastatic functions in esophageal cancer. Cancer Cell Int 20: 355, 2020.

39. Ma HF, Lv GX and Zhang DH: miR-381 mediates the development of head and neck squamous cell carcinoma via targeting STC2. Onco Targets Ther 13: 4485-4493, 2020.

40. Huo X, Han S, Wu G, Latchoumanin O, Zhou G, Hebbard L, George $\mathrm{J}$ and Qiao L: Dysregulated long noncoding RNAs (lncRNAs) in hepatocellular carcinoma: Implications for tumorigenesis, disease progression, and liver cancer stem cells. Mol Cancer 16: 165, 2017.

41. Zhang H, Xu HB, Kurban E and Luo HW: LncRNA SNHG14 promotes hepatocellular carcinoma progression via H3K27 acetylation activated PABPC1 by PTEN signaling. Cell Death Dis 11: 646, 2020

42. He H, Wang Y, Ye P, Yi D, Cheng Y, Tang H, Zhu Z, Wang X and Jin S: Long noncoding RNA ZFPM2-AS1 acts as a miRNA sponge and promotes cell invasion through regulation of miR-139/GDF10 in hepatocellular carcinoma. J Exp Clin Cancer Res 39: 159, 2020

43. Yin D, Hua L, Wang $\mathrm{J}$ and Liu Y: Long non-coding RNA DUXAP8 facilitates cell viability, migration, and glycolysis in non-small-cell lung cancer via regulating HK2 and LDHA by inhibition of miR-409-3p. Onco Targets Ther 13: 7111-7123, 2020.

44. Meng Q, Li Z, Pan J and Sun X: Long noncoding RNA DUXAP8 regulates proliferation and apoptosis of ovarian cancer cells via targeting miR-590-5p. Hum Cell 33: 1240-1251, 2020.
45. Xu LJ, Yu XJ, Wei B, Hui HX, Sun Y, Dai J and Chen XF: Long non-coding RNA DUXAP8 regulates proliferation and invasion of esophageal squamous cell cancer. Eur Rev Med Pharmacol Sci 22: 2646-2652, 2018.

46. Lin MG, Hong YK, Zhang Y, Lin BB and He XJ: Mechanism of lncRNA DUXAP8 in promoting proliferation of bladder cancer cells by regulating PTEN. Eur Rev Med Pharmacol Sci 22: 3370-3377, 2018.

47. Yang L, Mu Y, Cui H, Liang Y and Su X: MiR-9-3p augments apoptosis induced by $\mathrm{H} 2 \mathrm{O} 2$ through down regulation of Herpud 1 in glioma. PLoS One 12: e0174839, 2017.

48. Ding Y, Pan Y, Liu S, Jiang F and Jiao J: Elevation of MiR-9-3p suppresses the epithelial-mesenchymal transition of nasopharyngeal carcinoma cells via down-regulating FN1, ITGB1 and ITGAV. Cancer Biol Ther 18: 414-424, 2017.

49. Cai H, Yang X, Gao Y, Xu Z, Yu B, Xu T, Li X, Xu W, Wang X and Hua L: Exosomal MicroRNA-9-3p secreted from BMSCs downregulates ESM1 to suppress the development of bladder cancer. Mol Ther Nucleic Acids 18: 787-800, 2019.

50. Yang S, Cai H, Hu B and Tu J: LncRNA SAMMSON negatively regulates miR-9-3p in hepatocellular carcinoma cells and has prognostic values. Biosci Rep 39: BSR20190615, 2019.

51. Tang J, Li Y, Liu K, Zhu Q, Yang WH, Xiong LK and Guo DL: Exosomal miR-9-3p suppresses HBGF-5 expression and is a functional biomarker in hepatocellular carcinoma. Minerva Med 109: 15-23, 2018.

52. Militello G, Weirick T, John D, Döring C, Dimmeler S and Uchida S: Screening and validation of lncRNAs and circRNAs as miRNA sponges. Brief Bioinform 18: 780-788, 2017.

53. Olgun G, Sahin O and Tastan O: Discovering lncRNA mediated sponge interactions in breast cancer molecular subtypes. BMC Genomics 19: 650, 2018.

54. Yoon JH, Abdelmohsen $\mathrm{K}$ and Gorospe M: Functional interactions among microRNAs and long noncoding RNAs. Semin Cell Dev Biol 34: 9-14, 2014

55. Li DS, Ainiwaer JL, Sheyhiding I, Zhang Z and Zhang LW: Identification of key long non-coding RNAs as competing endogenous RNAs for miRNA-mRNA in lung adenocarcinoma. Eur Rev Med Pharmacol Sci 20: 2285-2295, 2016.

56. Lin P, Wen DY, Li Q, He Y, Yang H and Chen G: Genome-wide analysis of prognostic lncRNAs, miRNAs, and mRNAs forming a competing endogenous RNA Network in hepatocellular carcinoma. Cell Physiol Biochem 48: 1953-1967, 2018.

57. Schwartz MA and Ginsberg MH: Networks and crosstalk: Integrin signalling spreads. Nat Cell Biol 4: E65-E68, 2002.

58. Han X, Wang X, Zhao B, Chen G, Sheng Y, Wang W and Teng M: MicroRNA-187 inhibits tumor growth and metastasis via targeting of IGF-1R in hepatocellular carcinoma. Mol Med Rep 16: 2241-2246, 2017.

This work is licensed under a Creative Commons Attribution-NonCommercial-NoDerivatives 4.0 International (CC BY-NC-ND 4.0) License. 